\title{
Avaliação da maturidade pulmonar de cabritos nascidos a termo e prematuros
}

[Evaluation of lung maturity of term and premature kid]

F.L.F. Feitosa ${ }^{1}$, G.I. Braga ${ }^{2}$, L.C.N. Mendes ${ }^{1}$, J.F. Alcindo ${ }^{2}$, N.C. Souza ${ }^{2}$, F. Bovino ${ }^{3}$, T.A. Trein' 2 , J.T. Trevizan' ${ }^{2}$, R.S. Baptista ${ }^{2}$

${ }^{1}$ Universidade Estadual Paulista - Unesp - Faculdade de Medicina Veterinária, Araçatuba, SP

${ }^{2}$ Aluno de pós-graduação - Unesp - Faculdade de Medicina Veterinária, Araçatuba, SP

${ }^{3}$ Faculdade de Ciências Agrárias de Andradina - Fundação Educacional de Andradina - Andradina, SP

\section{RESUMO}

Avaliou-se a maturidade pulmonar de cabritos no líquido amniótico de suas mães pela coloração de Shor, pelo azul de Nilo e pela contagem de corpos lamelares, bem como a vitalidade e os níveis de glicose e lactato séricos em cabritos nascidos a termo e prematuros. Para tanto, foram utilizados 32 cabritos, divididos em três grupos, a saber: grupo I: cabritos nascidos de cesarianas com 149 dias de gestação; grupo II: cabritos nascidos de cesarianas com 143 dias de gestação; e grupo III: cabritos nascidos de cesarianas com 143 dias de gestação, oriundos de mães que receberam, por via intramuscular, $20 \mathrm{mg} /$ cabra de dexametasona, 36 horas antes da cirurgia eletiva. A coloração de Shorr e a contagem de corpos lamelares demonstraram ser métodos diagnósticos promissores para a avaliação da maturidade pulmonar em neonatos caprinos. Contudo, a administração de dexametasona nas cabras no período antenatal não influenciou a maturidade fetal. Constatou-se, entretanto, que a avaliação física do paciente, logo após o nascimento, também se mostra fundamental no que tange à percepção da vitalidade e da viabilidade de cabritos recém-nascidos.

Palavras-chave: maturidade fetal, líquido amniótico, neonatologia, caprinos

\begin{abstract}
Pulmonary maturity of goats in their amniotic fluid was evaluated by Shor, Nile blue staining, and lamellar body count, as well as vitality and serum glucose and lactate levels in term and premature goats. Twentyfour kids were divided into three groups: group I: comprised of eight animals born by cesarean section with 149 days of gestation; group II: comprised of eight animals born by cesarean section with 143 days of gestation; and group III: comprised of eight animals born by cesarean section with 143 days of gestation, in which the does received intramuscular dexamethasone (20mg/goat) 36 hours prior to elective cesarean section. Shorr staining and lamellar body count have shown to be promising diagnostic methods for the assessment of lung maturity in goat neonates. However, the administration of dexamethasone to goats during antenatal period did not influence fetal maturity. It was verified that the physical evaluation of the patient, shortly after birth, is fundamental for the perception of vitality and viability of newborn goats.
\end{abstract}

Keywords: fetal maturity, amniotic fluid, neonatology, goats

\section{INTRODUÇÃO}

Na medicina veterinária, a neonatologia mostra-se como recente especialização, e estudos relativos à fisiologia e à assistência neonatal nas diversas espécies ainda são escassos, o que contribui para o baixo grau de treinamento técnico, acarretando ineficiência da monitorização e da reanimação neonatal usualmente adequadas.

Dentro desse contexto, é de importância para o clínico avaliar o grau de maturidade pulmonar fetal, pois qualquer alteração na formação de qualquer estrutura pulmonar ou a simples

Recebido em 16 de maio de 2019

Aceito em 11 de dezembro de 2019

E-mail: francisco.feitosa@unesp.br 
ocorrência de recém-nascidos prematuros pode acarretar problemas respiratórios, como a síndrome da angústia respiratória (SAR). Evitar o nascimento prematuro constitui a principal medida preventiva para reduzir os riscos e as sequelas da morbidade respiratória neonatal (Taborda et al., 1998).

A avaliação da maturidade fetal pelo líquido amniótico (LA) é escassa na medicina veterinária, sendo inexistente na espécie caprina. A importância do presente estudo é demonstrada na rotina prática, quando há necessidade de interrupção ou de indução do parto, devido ao comprometimento materno, como a hidropsia dos envoltórios fetais e, mais comumente, a toxemia da prenhez, ou, ainda, quando há recém-nascidos oriundos de partos distócicos e/ou prematuros. Assim, o objetivo do presente estudo foi avaliar a eficiência dos testes de Clements, da coloração de azul de Nilo e da hematoxilina-Shorr, bem como da contagem de corpos lamelares no líquido amniótico de cabras para a vitalidade e a viabilidade de cabritos. Também foram determinados os níveis glicêmicos e de lactato de cabritos nascidos a termo e prematuros.

\section{MATERIAL E MÉTODOS}

O presente estudo foi aprovado pelo Comitê de Ética Animal da Faculdade de Medicina Veterinária, Unesp/Araçatuba (Ceua, n ${ }^{\circ}$ 201400473). Foram utilizados 32 cabritos mestiços da raça Boer. A avaliação prévia das gestações das respectivas cabras criadas sob as mesmas condições de manejo permitiu constatar que o tempo para a parição variava de 153 a 150 dias de gestação, ou seja, os animais prematuros nasceram com, no mínimo, 10 dias antes do período gestacional normal.

Os cabritos foram alocados em três grupos experimentais, a saber: grupo I (controle): cabritos nascidos por cesarianas realizadas aos 149 dias de gestação; grupo II: cabritos nascidos por cesarianas realizadas aos 143 dias de gestação; e grupo III: cabritos nascidos por cesarianas realizadas aos 143 dias de gestação, cujas mães receberam 20mg de dexametasona (Azium $^{\circledR}, \quad$ Schering-Plough), por via intramuscular, 36 horas antes das cirurgias eletivas. A comprovação e o acompanhamento das gestações foram procedidos pela observação da cobertura natural das fêmeas e por meio de ultrassonografia.

Empregou-se a anestesia local com bloqueio paravertebral proximal nos ramos nervosos das vértebras T13, L1 e L2, utilizando-se cloridrato de lidocaína (Xylestesin ${ }^{\circledR}$ 2\%, Cristália). Após a exposição placentária, rompiam-se as membranas corioalantoideana e amniótica, delicadamente, e retirava-se a maior quantidade de LA possível, com auxílio de agulha 40×12 acoplada à seringa de $20 \mathrm{~mL}$.

A avaliação das maturidades fetal e pulmonar foi realizada pelos testes de Clements et al. (1972) e Barreto (2006), pela contagem de corpos lamelares e pela citologia com azul de Nilo a $0,1 \%$. Realizou-se, concomitantemente, o método de hematoxilina-Shorr, utilizado por Moya (2005), como teste alternativo. Testou-se, também, nova modificação da técnica proposta por Clements et al. (1972).

No teste de Clements, modificado pelos autores do presente estudo, foram realizadas diferentes diluições, a fim de, possivelmente, obterem-se resultados confiáveis para a espécie caprina, como se segue : tubo $1(2,0 \mathrm{~mL}$ de $\mathrm{LA}+0,5 \mathrm{~mL}+$ etanol $95 \%)$; tubo $2(1,75 \mathrm{~mL}$ de $\mathrm{LA}+0,5 \mathrm{~mL}$ de etanol $95 \%)$; tubo $3(1,50 \mathrm{~mL}$ de $\mathrm{LA}+0,5 \mathrm{~mL}$ de etanol $95 \%)$; e tubo $4(1,25 \mathrm{~mL}$ de $\mathrm{LA}+0,5 \mathrm{~mL}$ de etanol 95\%), sendo elas classificadas como (1) negativo ou "imaturo" - não persistência de bolhas no primeiro tubo ou somente nos dois primeiros tubos e ausente no terceiro; (2) inconclusivo formação de halo até o terceiro tubo, ou presença de halo muito fraco nos quatro tubos; e (3) positivo ou "maduro" - caracterizada por espuma estável até o quarto tubo.

A contagem de corpos lamelares (CCL) foi processada no canal de plaquetas em analisadores hematológicos, necessitando-se de $0,5 \mathrm{~mL}$ de LA por amostra. $\mathrm{O}$ equipamento foi calibrado com os parâmetros utilizados para a contagem de plaquetas para murídeos e coelho. As amostras descongeladas em recipientes contendo água e gelo reciclável foram diluídas de acordo com Machado (2008), sendo homogeneizadas durante dois minutos e testadas por sistema automatizado BC-2800 VET Mindray ${ }^{\circledR}$, no canal de plaquetas, em que se considerou a medida volumétrica e a distribuição de tamanho e se comparou a curva logarítmica, a tecnologia de fluxo de arraste, 
conforme Fakhoury et al. (1994), Carrillo et al. (1997) e Lewis et al. (1999), três vezes em cada murídeo e coelho, obtendo-se, a partir de então, a média dos valores alcançados. Encaminhou-se um total de seis amostras de LA, sendo duas de cada grupo (com dois mililitros, aproximadamente) para processamento e posterior análise por microscopia eletrônica (JEOL JEM 100 CX), com o intuito de se avaliar a presença de corpos lamelares.

Para avaliação da vitalidade dos cabritos, utilizouse o escore Apgar, modificado por Born (1981). As mensurações da glicemia e da concentração plasmática de lactato foram realizadas imediatamente antes de o cordão umbilical ser clampeado e posteriormente a isso, nos momentos anteriormente descritos, em glicosímetro (One Touch Ultra II ${ }^{\circledR}$, Johnson \& Johnson) e lactímetro (Accutrend Plus ${ }^{\circledR}$, Roche), respectivamente, seguindo-se as recomendações dos fabricantes.

Os dados foram submetidos à análise de variância, considerando-se delineamento inteiramente ao acaso, com medidas repetidas no tempo (três grupos e seis momentos). As médias foram comparadas pelo teste de Tukey, no nível de significância de 5\%. Tabelas com a mediana eram analisadas empregando-se o teste de KruskalWallis, para se compararem os grupos em cada momento, e o teste de Friedman, para se compararem os momentos em cada grupo, seguidos do teste de Dunn, para comparações múltiplas. Os dados foram testados quanto à normalidade dos erros e à homogeneidade de variâncias. As variáveis Apgar e teste de Clements foram analisadas pelo teste exato de Fisher.

O teste ANOVA foi utilizado para avaliação entre os tempos dentro de cada grupo em algumas variáveis, bem como para análise de medidas repetidas no tempo. As estatísticas foram consideradas significativas quando $\mathrm{P}<0,05$. As análises estatísticas foram efetuadas empregandose o programa SAS (Statistical Analysis System SAS Institute Inc. The SAS System, release 9.3., SAS (Statistical..., 2016).

\section{RESULTADOS E DISCUSSÃO}

Foram acompanhados os nascimentos de 32 animais oriundos de cirurgias eletivas em 20 cabras. A avaliação física do recém-nascido, logo após o nascimento, gera informações sobre possíveis infecções sistêmicas, alterações localizadas e/ou adquiridas e existência de dispneia (Vaala e House, 2006). Não houve mortalidade dos recém-nascidos, demonstrando que cabritos prematuros oriundos de cesarianas eletivas, aos 143 dias gestação, são viáveis. Os valores do escore Apgar modificado (7 a 8) não demonstraram diferenças significativas entre os grupos, classificando-se como boa a ótima vitalidade. Os resultados mostraram-se compatíveis aos obtidos em cabritos nascidos a termo por Camargo (2010), e em cordeiros por Bovino (2015), os quais verificaram vitalidade normal (pontuação 7 a 8) nos primeiros minutos de vida $(84,00 \%$ e $93,75 \%)$, respectivamente.

Denotou-se que, ao nascimento, um animal pertencente ao grupo I e outro ao grupo II receberam a pontuação de 0-3, a qual foi correlacionada à baixa vitalidade, por esses animais terem baixo peso ao nascimento. Esses cabritos necessitaram de suporte ventilatório logo após o nascimento e da administração de colostro via mamadeira, vindo a atingir escore moderado nos momentos posteriores. Os dados deste estudo discordam de algumas descrições em relação ao aumento da pontuação do escore Apgar, com o passar do tempo dos neonatos, haja vista que se obteve escore moderado ao longo do período de avaliação (até 48 horas). Essa diminuição não significa, necessariamente, declínio da atividade física ou da viabilidade dos recém-nascidos.

Por vezes, tal fato decorre da "indiferença" ou do fato de se tornarem menos "responsivos" ou intuitivamente "adaptados" aos estímulos que são realizados com o passar do tempo, principalmente no que concerne ao reflexo de resposta à água fria. Dados semelhantes foram encontrados por Ávila (2013) e Bovino (2015), que constataram diminuição da porcentagem do escore Apgar, aos 15 e 60 minutos após o nascimento. A vitalidade moderada pode ainda estar relacionada ao tipo de parto. Segundo Silva (2008), neonatos nascidos por cesarianas apresentavam escore inferior aos demais indivíduos nascidos de parturições normais. Entretanto, ficou evidenciado, no presente trabalho, que os cabritos não sofreram influência em sua vitalidade em decorrência da cirurgia eletiva e dos fármacos utilizados. No entanto, pontuação abaixo de seis necessita de maior atenção por parte do examinador, já que reflete animais com dificuldade de adaptação à vida extrauterina. 
O líquido amniótico apresentava-se claro e transparente, com viscosidade variável. Em três deles verificou-se presença de mecônio. Os testes descritos por Clements et al. (1972) e Barreto (2006) não evidenciaram resultado positivo preditivo para maturidade pulmonar. Diante de tais dados, propôs-se a realização de nova adequação da técnica, realizando-se várias diluições. Analisando-se os dados obtidos, verificou-se que $12,5 \%$ (grupo I), 37,5\% (grupo II) e $50 \%$ (grupo III) das amostras de líquido amniótico apresentavam-se negativas. Os resultados inconclusivos ficaram em 37,5\%, $37,5 \%$ e $50 \%$ nos grupos I, II e III, respectivamente, evidenciando que os métodos anteriormente descritos por Clements et al. (1972) e Barreto (2006), bem como o adaptado no presente trabalho, não se mostraram satisfatórios para tal finalidade.

Em virtude do elevado custo, apenas seis amostras de líquido amniótico foram submetidas à análise por microscopia eletrônica, visando determinar algumas características dos corpos lamelares (CL). Para avaliar o tamanho dessas estruturas, utilizou-se o programa Image $\mathbf{J}^{\circledR}$ (Fig. 1); a média obtida foi de $0,15 \mu \mathrm{m}$, sendo, portanto, de menor tamanho em relação àqueles obtidos no líquido

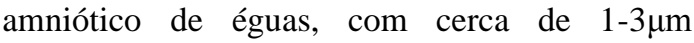
(Castagnetti et al., 2007). No presente trabalho, após diversas tentativas de filtragem das amostras de LA para separação do muco, não se obtiveram resultados confiáveis devido à alta viscosidade, já que grande parte do muco das amostras não proporcionou contagem no analisador automático. Decidiu-se, portanto, diluir as amostras de líquido amniótico com ditiotreitol, de acordo com Machado (2008), conseguindo-se, com isso, proceder ao teste no analisador automático. No momento da passagem das amostras de líquido amniótico pelo analisador automático BC-2800 VET Mindray ${ }^{\circledR}$, constatou-se, também, que, quando o aparelho era ajustado para leitura de componentes sanguíneos de murídeos e coelho, os valores eram mais facilmente obtidos, possivelmente, porque os ajustes foram feitos para a leitura de estruturas menores, ficando condizentes, portanto, com o pequeno tamanho dos corpos lamelares $(0,15 \mu \mathrm{m})$ encontrados no líquido amniótico de cabras. Denotou-se variação de 8.000 a $83.000 / \mu \mathrm{L}$ na quantidade de corpos lamelares presentes no líquido amniótico de cabras. Os animais do grupo I tinham as maiores concentrações nas três calibrações (ratos, camundongos e coelhos) avaliadas. Em contraposição, os animais do grupo III obtiveram os menores valores nos três modos.

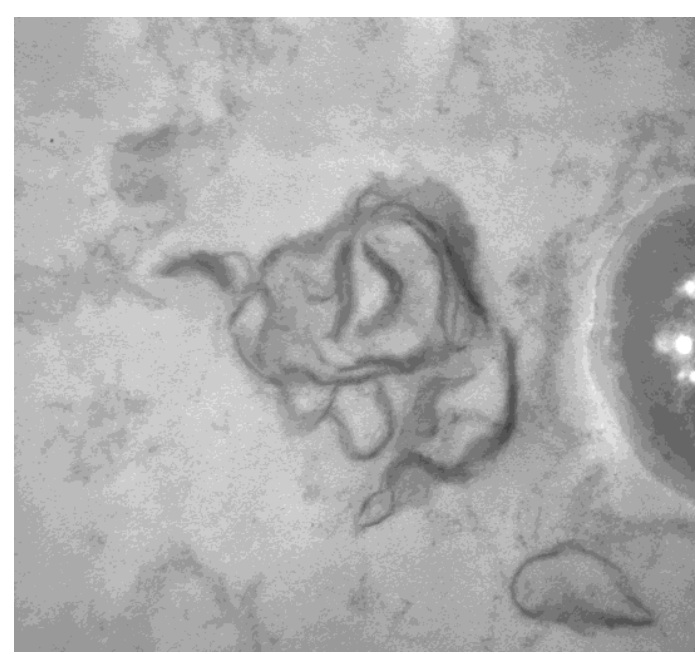

Figura 1. Corpo lamelar presente no líquido amniótico de cabra, evidenciando suas lâminas concêntricas, representadas por camada delgada de diâmetros reduzidos, acompanhada por um centro em sua estrutura.

Observou-se, na coloração por azul de Nilo (Tab. 1), elevado percentual de células cianofílicas em todas as amostras do LA, mesmo naquelas provenientes de cabras com período gestacional normal (grupo 1), sendo que a porcentagem de células orangeofílicas não ultrapassou 3\% em quaisquer dos grupos estudados. Soma-se a isso a subjetividade na execução do teste e a interpretação de seus resultados, haja vista que algumas células coravam-se de verde-claro, o que destoava completamente das variações preconizadas (azul ou laranja), contrariando o descrito por Souza et al. (2000), para a espécie ovina, e por Bongso e Basrur (1975), para a bovina, que obtiveram resultados satisfatórios com a referida técnica. Os dados do presente trabalho assemelham-se aos encontrados por Moya (2005), que não constatou resultados consistentes com o uso da coloração do azul de Nilo para a espécie bovina, e por Martins e Prestes (2003) sobre a avaliação do grau de maturidade de neonatos caninos.

Contudo, na análise das células do líquido amniótico pela coloração de hematoxilina-Shorr (Tab. 1), os resultados mostraram-se mais consistentes em relação ao grau de maturidade apresentado pelos cabritos, tendo em vista 
maiores porcentagens de células orangeofilicas encontradas $(77 \%, 69,4 \%$ e $79,1 \%$, nos grupos I, II e III, respectivamente). As porcentagens de células cianofílicas foram de $23 \%, 30,6 \%$ e 20,9\% nos grupos I, II e III, respectivamente, o que demonstra melhor maturidade nos cabritos nascidos com período gestacional normal e oriundos de mães que receberam corticoterapia 36 horas antes da cirurgia eletiva. No teste em questão, decidiu-se não utilizar qualquer tipo de centrifugação para confecção das lâminas coradas pelo método hematoxilina-Shorr. Foi constatado que, quando as amostras não eram centrifugadas, as lâminas apresentavam maior número celular, em virtude da obtenção da porção mais mucoide do líquido amniótico. Ao se realizar a centrifugação, o muco é separado, havendo, portanto, maior retenção de parte das células presentes no líquido amniótico.

Tabela 1. Média $(\bar{x})$ e desvio-padrão $(\mathrm{S})$ dos percentuais de células cianofílicas e orangeofílicas coradas pelos métodos de azul de Nilo e Hematoxilina-Shorr no líquido amniótico de 20 cabras submetidas ou não à corticoterapia antenatal

\begin{tabular}{ccccc}
\hline \multirow{2}{*}{ Grupos } & \multicolumn{2}{c}{ Coloração Azul de Nilo } & \multicolumn{2}{c}{ Hematoxilina-Shorr } \\
\cline { 2 - 5 } & Cianofílicas & Orangeofílicas & Cianofílicas & Orangeofílicas \\
\hline I & $97,1 \pm 1,6^{*}$ & $2,9 \pm 1,6^{*}$ & $23,0 \pm 5,1 \mathrm{~b}$ & $77,0 \pm 5,1 \mathrm{a}$ \\
II & $98,3 \pm 0,9^{*}$ & $1,7 \pm 0,9^{*}$ & $30,6 \pm 4,7 \mathrm{a}$ & $69,4 \pm 4,7 \mathrm{~b}$ \\
III & $98,2 \pm 1,5^{*}$ & $1,8 \pm 1,5^{*}$ & $20,9 \pm 1,9 \mathrm{~b}$ & $79,1 \pm 1,9 \mathrm{a}$ \\
\hline
\end{tabular}

*P $<0,05$ em relação a técnica Hematoxilina Shorr

Médias seguidas de letras distintas, na coluna, diferem entre si pelo teste de Tukey $(\mathrm{P}<0,05)$

O período despendido entre a leitura das lâminas não ultrapassou 60 minutos, característica interessante, tendo em vista que a rapidez para a obtenção de indícios sobre a maturidade fetal é relevante do ponto de vista clínico, por propiciar rápida decisão sobre a conduta terapêutica e o procedimento apropriado para a reanimação. Os animais prematuros do grupo II, constituído por cabritos prematuros cujas mães não receberam corticoterapia, apresentaram o maior índice de células cianofílicas, indicando, dessa forma, menor maturidade pulmonar. Assim, a técnica mostrou-se confiável para avaliação da maturidade pulmonar de cabritos, bem como exequível, por necessitar apenas de lâminas, dos constituintes da coloração e de microscópio para sua realização.

A glicose, considerada como o mais importante substrato para a oxidação respiratória, é vital para o metabolismo cerebral e a lactação. O nível de glicose sanguínea pode indicar falhas na homeostase, como no caso de cetose (Gonzalez Filho e Sheffer, 2002). A compilação dos dados obtidos do presente trabalho é deveras complicada em virtude da ausência ou carência de informações de valores referenciais dos níveis séricos para a categoria etária. Araújo e Silva (2008) consideraram teores de glicose em caprinos entre dois e quatro anos variando de 45,2 a $51,5 \mathrm{mg} / \mathrm{dL}$. Do ponto de vista individual, constatou-se, no grupo III, que o sangue obtido na veia umbilical continha menor valor de glicose do que o obtido da veia jugular imediatamente após o nascimento.

Levando-se em consideração os animais do grupo I, que nasceram em período gestacional condizente para a espécie, observou-se variação entre 28 e 46mg/dL logo após o nascimento, ou seja, com aporte glicêmico satisfatório, mesmo antes de terem acesso ao colostro. Os animais ingeriam colostro até duas horas de nascidos. Os menores níveis de glicose foram obtidos cerca de uma hora após o nascimento, com posterior elevação da glicemia (Tab. 2) ao longo do tempo, particularmente às 48 horas de vida, em decorrência da ingestão da secreção láctea e do adequado manejo alimentar adotado para as cabras durante o período gestacional.

Esses dados concordam com os descritos por Gregory et al. (2009), que relataram valores glicêmicos médios em cabritos, ao nascimento, de 29,70; aos 30 minutos de 53,96; a uma hora, de 54,51; e de 88,82 e de 104, 92 (mg/dL), às 24 e 48 horas de vida. Mostram-se, entretanto, contraditórios à afirmação de Andrade (2002), que ressaltou que prematuros não possuem reservas adequadas de glicose devido à carência de tempo hábil para prover estoques energéticos satisfatórios. 
Tabela 2. Mediana (Md), valores mínimos (Mín) e máximos (Máx) dos níveis séricos de glicose (mg/dL) obtidos nas veias umbilical e jugular de 32 cabritos nascidos por cesariana, aos 149 dias (grupo I) e aos 143 dias (grupos II e III), sem e com a administração de dexametasona em suas mães

\begin{tabular}{|c|c|c|c|c|c|c|c|c|c|}
\hline \multirow{3}{*}{ Momentos } & \multicolumn{9}{|c|}{ Grupos } \\
\hline & \multicolumn{3}{|c|}{ I } & \multicolumn{3}{|c|}{ II } & \multicolumn{3}{|c|}{ III } \\
\hline & $\mathrm{n}$ & $\mathrm{Md}$ & Mín - Máx & $\mathrm{n}$ & Md & Mín - Máx & $\mathrm{n}$ & $\mathrm{Md}$ & Mín - Máx \\
\hline $\begin{array}{c}\text { Veia } \\
\text { umbilical }\end{array}$ & 8 & $34^{\mathrm{AB}}$ & $20-40$ & 8 & $26,5^{\mathrm{Aa}}$ & $20-38$ & 8 & $36^{\mathrm{Ba}}$ & $24-76$ \\
\hline $\mathrm{M}_{0}$ & 8 & 31 & $28-46$ & 8 & 25,5 & $20-79$ & 8 & 66 & 20-111 \\
\hline $\mathbf{M}_{15}$ & 8 & $24^{\mathrm{a}}$ & $20-74$ & 8 & $26^{\mathrm{a}}$ & $20-62$ & 8 & 62 & $20-155$ \\
\hline $\mathbf{M}_{30}$ & 8 & 31,5 & $21-62$ & 8 & $24,5^{\mathrm{a}}$ & $20-41$ & 8 & 46 & $20-75$ \\
\hline $\mathbf{M}_{60}$ & 8 & $22^{\mathrm{Aba}}$ & $20-73$ & 8 & $22^{\mathrm{Aa}}$ & $20-49$ & 8 & $58^{\mathrm{B}}$ & 24-202 \\
\hline $\mathbf{M}_{24}$ & 8 & $115^{\mathrm{b}}$ & $38-138$ & 8 & $93,5^{\mathrm{b}}$ & 24-179 & 8 & 125 & $62-168$ \\
\hline $\mathrm{M}_{48}$ & 8 & $131,5^{\mathrm{b}}$ & $120-141$ & 8 & $26,5^{\mathrm{b}}$ & $72-167$ & 8 & $145^{\mathrm{b}}$ & 71-185 \\
\hline
\end{tabular}

As medianas seguidas de letras diferentes, maiúscula na linha e minúscula na coluna, diferem entre si pelo teste de Kruskal-Wallis e pelo teste de Friedman, seguidos pelo pós-teste de Dunn $(\mathrm{P}<0,05)$. A ausência de letras implica que não há diferença estatística.

$\mathrm{Na}$ obstetrícia humana, o lactato desempenha papel central como marcador de sofrimentos fetal e neonatal. A presença de altos níveis de lactato promove a utilização de vias secundárias de oxigenação devido à hipóxia decorrente de eventos que ocorreram durante o parto (Groppetti et al., 2010). O teor de lactato não mostrou alteração antes do clampeamento do cordão umbilical aos níveis obtidos após o seu seccionamento. De acordo com dados presentes na Tab. 4, verificou-se que houve significância estatística entre os grupos no M48, em que o grupo I difere do III. Já em relação aos momentos, ocorreu diferença estatística no M60 em relação ao M48 nos cabritos do grupo I, verificando-se diminuição nos valores de lactato às 48 horas após o nascimento, possivelmente em virtude da ventilação respiratória satisfatória.
Constatou-se variação estatística nos cabritos do grupo II nos momentos M0 e M15 em relação ao M48, e no grupo III no M0 em relação aos M24 e M48. Ao contrário do esperado, os animais pertencentes ao grupo I não apresentaram os menores valores de lactato. $\mathrm{O}$ mesmo resultado foi observado nos demais grupos. A elevação dos níveis de lactato ao nascimento é fisiológica e deve-se à estimulação generalizada do sistema nervoso simpático, com liberação de adrenalina e cortisol, durante e após o nascimento, promovendo não somente o aumento de lactato como também do volume globular, da glicose, da frequência cardíaca e dos ácidos graxos livres, conforme proposto por Comline e Silver (1972).

Tabela 3. Mediana (Md), valores mínimos (Mín) e máximos (Máx) dos níveis séricos de lactato ( $\mu$ mol/L) obtidos nas veias umbilical e jugular de 32 cabritos nascidos por cesariana, aos 149 dias (grupo I) e aos 143 dias (grupos II e III), sem e com a administração de dexametasona em suas mães

\begin{tabular}{|c|c|c|c|c|c|c|c|c|c|}
\hline \multirow{3}{*}{ Momentos } & \multicolumn{9}{|c|}{ Grupos } \\
\hline & \multicolumn{3}{|r|}{$\mathrm{I}$} & \multicolumn{3}{|c|}{ II } & \multicolumn{3}{|c|}{ III } \\
\hline & $\mathrm{n}$ & $\mathrm{Md}$ & Mín-Máx & $\mathrm{n}$ & $\mathrm{Md}$ & Mín - Máx & $\mathrm{n}$ & $\mathrm{Md}$ & Mín - Máx \\
\hline Veia umbilical & 8 & 8,85 & $9-11,2$ & 8 & 7,25 & $5,5-9$ & 8 & 8,05 & $3,8-17,6$ \\
\hline $\mathrm{M}_{0}$ & 8 & 8,45 & $7,1-12,6$ & 8 & $9,05^{\mathrm{a}}$ & $6,5-12,7$ & 8 & $10,25^{\mathrm{a}}$ & $7,3-21$ \\
\hline $\mathbf{M}_{15}$ & 8 & 9,35 & $5,7-12,7$ & 8 & $8,95^{\mathrm{a}}$ & $6,3-13,8$ & 8 & 7,6 & $5,5-16,7$ \\
\hline $\mathrm{M}_{30}$ & 8 & 8,8 & $5,2-13,2$ & 8 & 8,4 & $5,2-14,9$ & 8 & 7,3 & $4,1-15,3$ \\
\hline $\mathrm{M}_{60}$ & 8 & $10,5^{\mathrm{a}}$ & $7,3-15,1$ & 8 & 8 & $4,3-14,6$ & 8 & 7,8 & $5,7-13,2$ \\
\hline $\mathrm{M}_{24}$ & 8 & 7,45 & $6-9,2$ & 8 & 5,2 & $4,2-11,7$ & 8 & $6,05^{\mathrm{b}}$ & $3,4-9,5$ \\
\hline $\mathrm{M}_{48}$ & 8 & $7,3^{\mathrm{Ab}}$ & $4,6-8,5$ & 8 & $4,05^{\mathrm{ABb}}$ & $3,4-10,6$ & 8 & $3,85^{\mathrm{Bb}}$ & $3-6,2$ \\
\hline
\end{tabular}

As medianas seguidas de letras diferentes, maiúscula na linha e minúscula na coluna, diferem entre si pelo teste de Kruskal-Wallis e pelo teste de Friedman, respectivamente, seguidos pelo pós-teste de Dunn $(\mathrm{P}<0,05)$. A ausência de letras implica que não há diferença estatística. 


\section{CONCLUSÕES}

O escore Apgar modificado demonstrou animais com vitalidade satisfatória; o método citológico pela coloração de hematoxilina-Shorr e a contagem de corpos lamelares forneceram resultados promissores para a avaliação da maturidade pulmonar; os níveis séricos de glicose apresentam-se mais baixos ao nascimento, elevando-se com o passar do tempo, em virtude da ingestão de colostro. As maiores concentrações de lactato foram obtidas logo após o nascimento, com gradativa redução ao longo das 48 horas de vida; a associação das referidas variáveis concomitantemente ao exame físico é ferramenta indispensável à percepção e detecção de animais com dificuldade respiratória (hipóxia) e adaptação ao meio extrauterino. A administração de dexametasona antes da realização da cirurgia eletiva não demonstra influência sobre a vitalidade dos recém-nascidos.

\section{AGRADECIMENTOS}

À Fundação de Amparo à Pesquisa do Estado de São Paulo (Fapesp), pelo financiamento do projeto (2012/21967-8) e pela concessão de bolsa de pesquisa (2012/21967-4).

\section{REFERÊNCIAS}

ANDRADE, S.M.A. Glicemia neonatal comparação dos resultados da determinação da glicemia em recém-nascidos através de amostra sérica venosa e amostra de sangue capilar. 2002. 91f. Dissertação (Mestrado em Medicina Pediátrica) Faculdade de Medicina, Universidade Federal do Rio Grande do Sul, Porto Alegre RS.

ARAÚJO, D.F.; SILVA, I.P. Valores de amilase, glicose, colesterol e triglicérides em soro de cabras de Mossoró, RN. Acta Vet. Bras., v.2, p.97-100, 2008.

ÀVILA, L.G. Avaliação clínico-laboratorial de cordeiros nascidos a termo e prematuros. 2013. $86 f$. Tese (Doutorado em Ciência Animal) Universidade Estadual Paulista, Faculdade de Medicina Veterinária, Araçatuba, SP.

BARRETO, C.S. Aspectos fisiológicos da maturidade fetal em cães. 2006. 74f. Tese (Doutorado) - Faculdade de Medicina Veterinária e Zootecnia, Universidade Estadual Paulista "Júlio de Mesquita Filho", Botucatu, SP.
BONGSO, T.A.; BASRUR, P.K. Prenatal diagnosis of sex in cattle by amniocentesis. Vet. Rec, v.8, p.124-126, 1975.

BORN, E. Untersuchungen uber den Einfluss der Schnittentbindung auf die Vitalitat neugeborener Kalber. 1981. 47f. These (Doutorado). Tierarztliche ochschule, Hannover, DE.

BOVINO, F. Abordagem clínica de cordeiros prematuros: avaliação de protocolos terapêticos emergenciais para estimulação $d a$ atividade respiratória. 2015. 82f. Tese (Doutorado em Ciência Animal) - Universidade Estadual Paulista, Faculdade de Medicina Veterinária, Araçatuba, SP.

CAMARGO, D.G. Avaliação do sistema Apgar (modificado por Born, 1981) e dos níveis de cortisolemia, glicemia e de gases sanguíneos em cabritos nascidos de partos eutócicos e de cesariana. 2010. 93f. Dissertação (Mestrado em Ciência Animal) - Universidade Estadual Paulista, Faculdade de Medicina Veterinária, Araçatuba, SP.

CARRILLO, J.B.; GARCIA, E.; LOZANO, C.G. Cuantificación de cuerpos lamelares en líquido amniótico: método de valoración de madurez pulmonar fetal. Ginecol. Obster. Mex. v.65, p.202206, 1997.

CASTAGNETTI, C.; MARIELLA, J.; SERRAZANETTI, G. P.; GRANDIS, C. et al. Evaluation Of Lung Maturity By Amniotic Fluid Analysis in equine neonate. Theriogenology, v.67, p.1455-1462, 2007.

CLEMENTS, J.A.; PLATZKER, A.C.G.; TIERNEY, D.F.; HOBEL, C.J. et al. Assessment of the risk of respiratory-distress syndrome by a rapid test for surfactant in amniotic fluid. Engl. J. Med., v.286, p.1077-1082, 1972.

COMLINE, R.S.; SILVER, M. The composition of foetal and maternal blood during parturition in the ewe. J. Physiol., v.222, p.233-256, 1972.

FAKHOURY, G.; DAIKOKU, N.H.; BENSER, J.; DUBIN, N. Lamellar body concentrations and the prediction of fetal pulmonary maturity. Am. J. Obstet. Gynecol. v.170, p.72-76, 1994.

GONZALEZ F.H.D.; SHEFFER, J.F.S. Perfil sanguíneo: ferramenta de análise clínica, metabólica e nutricional. In: AVALIAÇÃO METABÓLICONUTRICIONAL DE VACAS LEITEIRAS POR MEIO DE FLUIDOS CORPORAIS, 29, 2002, Gramado. Anais... Gramado: Felix / González, 2002. $72 \mathrm{p}$. 
GREGORY, L.; BARDESE, C.B.; BIRGEL JR., E.H.; MEIRA JR., E.B.S. et al. Lipidograma e glicemia de caprinos da raça Saanen, durante os primeiros dias de vida. Ars Vet., v.25, p.109-115, 2009

GROPPETTI, D.; PECILE, A.; DEL CARRO, A.P.; COPLEY, K. et al. Evaluation of newborn canine viability by means of umbilical vein lactate measurement, apgar score and uterine tocodynamometry. Theriogenology, v.20, p.11871196, 2010.

LEWIS, P.S.; LAURIA, M.R.; DZIECZKOWSKI, J.; UTTER, G.O. et al. Amniotic fluid lamellar body count: cost-effective screening for fetal lung maturity. Obstet. Gynecol., v.93, p.387-391, 1999.

MACHADO, L.U. Avaliação da produção e função surfatante através de testes de maturidade pulmonar no aspirado gástrico de recém-nascidos a termo com taquipneia transitória. 2008. 106f. Dissertação (Mestrado) - Faculdade de Medicina da PUCRS, Porto Alegre, RS.

MARTINS, L.R.; PRESTES, N.C. Ensaio sobre exame citologico do líquido amniotico de cadelas (Cannis familiaris) colhida no momento da cesariana. ARS Vet., p.294-299, 2003.

MOYA, C.F. Tipificação citológica do LA de bezerros oriundos de produção In Vitro, transferência de embrião e inseminação artificial no momento do parto. 2005. 64f. Dissertação (Mestrado) - Faculdade de Medicina Veterinária e Zootecnia, Universidade Estadual Paulista "Júlio de Mesquita Filho", Botucatu, SP.
SILVA, L.C.G. Parâmetros clínicos, hemogasométricos e radiográficos para avaliação respiratória de neonatos caninos nascidos em eutocia ou cesariana eletiva. 2008. 75f. Dissertação (Mestrado) - Faculdade de Medicina Veterinária e Zootecnia, Departamento Reprodução Animal, Universidade de São Paulo, São Paulo, SP.

SOUZA, C.; PRESTES, N.C.; LOPES, R.S. determinação da maturidade fetal através da análise de parâmetros físicos e bioquímicos do fluido amniótico de ovinos (Ovis aries), colhidos em abatedouro. In: CONGRESSO BRASILEIRO DE MEDICINA VETERINÁRIA, 17., 2000, São Paulo. Anais... São Paulo: [s.n.], 2000. (Resumo).

STATISTICAL analysis system. Version 9.3. Cary: SAS Institute Inc., 2016.

TABORDA, W.; ALMEIDA, M.F.; MORON, A.F.; BERTINI, A.M. Avaliação da maturidade pulmonar em gestantes de alto risco. Rev. Bra. Ginecol. Obstetr., v.20, p.315-321, 1998.

VAALA, W.E.; HOUSE, J.K. Manifestações de doença no neonato. In: SMITH, B.P. Medicina interna de grandes animais. 3.ed. Barueri: Manole, 2006. p.319-381. 